\title{
Cutaneous metastases from colon carcinoma-An unusual presentation.
}

\author{
Sourav Mishra ${ }^{1}$, Rashmi Patnayak ${ }^{2 *}$ Amitabh Jena ${ }^{2}$ \\ ${ }^{1}$ Department of Medical Oncology, Cancer Institute, Tamil Nadu, India \\ ${ }^{2}$ Institute of Medical Sciences and Sum Hospital, Odisha, India
}

\begin{abstract}
Background: Cutaneous metastases from colorectal cancer is a rare event. It indicates widely spread disease with poor prognosis.

Case Report: A 40 years old male, diagnosed case of adenocarcinoma of the ascending colon, presented with multiple cutaneous nodules over the trunk, back and the legs. Histopathological examination of one of the cutaneous nodule showed deposits of signet ring cell carcinoma.

Conclusion: Multiple cutaneous metastasis from signet ring cell carcinoma is uncommon. This entity should be recognized in patients of colonic primary as it depicts wide spread disease with poor prognosis.
\end{abstract}

Keywords: Cutaneous metastasis, Colon carcinoma, Signet ring cell carcinoma.

Accepted on November 22, 2018

\section{Introduction}

It is extremely rare to encounter cutaneous metastases from colorectal cancer. They often appear several years after diagnosis or resection of the primary tumor. Presence of cutaneous metastasis indicates poor prognosis and wide spread disease [1,2]. The present case is one such case who had multiple cutaneous metastasis of signet ring cell carcinoma from a colonic primary.

\section{Case Report}

A 40 years old male was diagnosed as adenocarcinoma of the ascending colon and was treated with chemotherapy and hemicolectomy in 2013. He was initially treated with eight cycles of Capeox to which he had partial response. He was then put on Capecitabine maintenance. He was in remission till march 2015, when he presented with multiple cutaneous nodules spread on the skin over the trunk, back and the legs. (Figures 1A and B) These lesions were erythematous, firm and nontender. Biopsy from one of the lesions was done and the histopathology was reported as metastatic signet ring cell adenocarcinoma. The cells were showing positivity for Alcian blue stain (Figures 2A and B). His colonic primary was still in remission and there were no other sites of metastases. He was started on second line chemotherapy (Capiri) following which his skin lesions showed some response and no new lesions developed subsequently. Soon after this the patient was lost to follow up.

\section{Discussion}

Cutaneous metastases of cancer are rare, occurring in about $1.3 \%$ of cases at the time of presentation of the primary tumor [1]. These metastases have also been associated with wide spectrum of malignancies including that from cancers of gastric, esophageal, breast, ovarian, hematologic, laryngeal, palatine-tonsillar, pancreatic, parotid, thyroid, uterine and various other sites [1-6]. In colorectal carcinoma, cutaneous metastases are rarely encountered. It accounts for $2.3 \%-6.0 \%$ of cases [3]. The most frequent site of cutaneous metastases of colon cancer is abdominal skin $[1,2]$. Other common cutaneous sites are the pelvis, back, chest, upper extremities, head and neck $[1,2]$. Clinically these lesions appear as painless, firm nodules or purple-red indurations termed as carcinoma erysipeloides [2,3,7]. Clinically they may be mistaken as lipomas or neurofibromas. Histopathology helps in establishing the correct diagnosis. The microscopic features of metastatic lesions resemble those of the primary tumor.

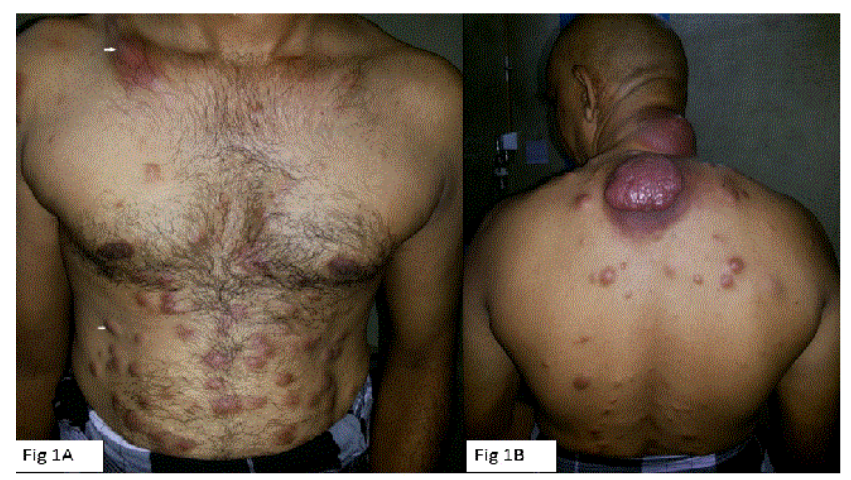

Figure 1. A) Clinical picture showing multiple erythematous cutaneous nodules over trunk (white arrows); B) Multiple erythematous cutaneous nodules over back

Majority of cutaneous metastases from large intestine tumors are well differentiated mucin-secreting adenocarcinomas [2]. The present case microscopically showed cutaneous metastasis of signet ring cell carcinoma.

The route of metastases of cutaneous metastases are lymphomatous, vascular, direct extension of tumor, surgical 
implantation and spread along embryonal remnants such as the urachus [1].

Skin metastases are a poor prognostic indicator of colon cancer because it usually reflects widespread disease $[1,2]$.

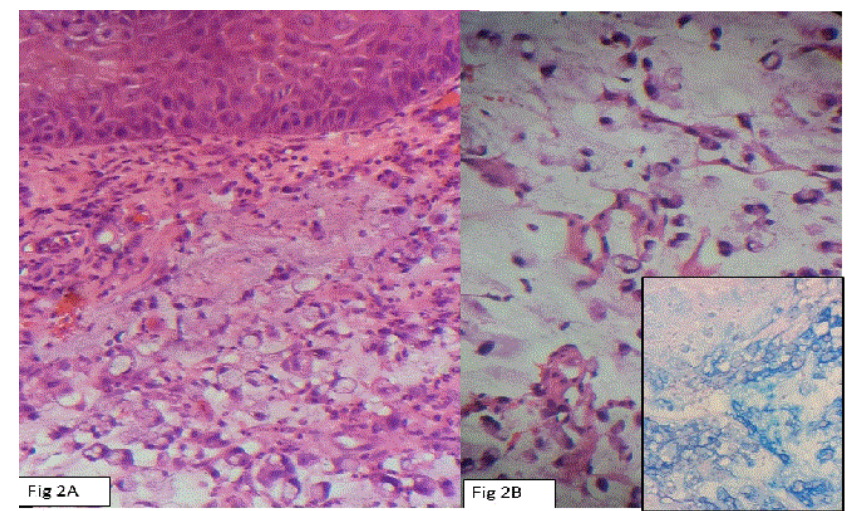

Figure 2. A) Histopathology of signet ring cell carcinoma present beneath the epidermis (Haematoxylin and eosin x 100); B) Mucin containing signet ring cells (Haematoxylin and eosin $x$ 400) Inset: Signet ring cells showing Alcian blue positivity for mucin

For isolated cutaneous lesion wide local excision is the preferred mode of treatment. Palliative treatment is the treatment of choice in extensive cutaneous metastases. Extensive tumor resection of those neoplasms can result in giant abdominal wall defects. There are no definite guidelines regarding the optimum chemotherapeutic regimens. Usual chemotherapy treatments include 5-fluorouracil, capecitabine, irinotecan, oxaliplatin and cisplatin. Combining irinotecan to bolus 5-FU (Fluorouracil), oxaliplatin to infusional 5FU and Leucovorin (LV) or infusional $5 \mathrm{FU} / \mathrm{LV}$ with irinotecan has increased survival to over 20 months [2,7]. Survival after diagnosis of cutaneous metastasis range from 1-34 months. The median survival for patients is 18 months $[1,2,7]$.

\section{Conclusion}

It is uncommon to encounter multiple cutaneous metastasis from primary signet ring cell carcinoma in colon. This entity needs to be considered in carcinoma colon patients presenting with cutaneous nodules. The prognosis is poor in these cases.

\section{References}

1. Wang DY, Ye F, Lin JJ, et al. Cutaneous metastasis: A rare phenomenon of colorectal cancer. Ann Surg Treat Res. 2017;93:277-80.

2. Nesseris I, Tsamakis C, Gregoriou S, et al. Cutaneous metastasis of colon adenocarcinoma: Case report and review of the literature. An Bras Dermatol. 2013;88:56-8.

3. Zanwar VG, Pawar SV, Jain SS, et al. Cutaneous lesions in colorectal carcinoma: A rare presentation. Intest Res. 2016;14:102-3

4. Hari K, Jena A, Chowhan AK, et al. The unnoticed umbilical nodule of ovarian malignancy with pseudomyxomaperitonei: A rare presentation. Indian $\mathrm{J}$ Cancer. 2015;52:705-7.

5. Narayana MA, Patnayak R, Rukmangadha $N$, et al. Cutaneous metastasis of transitional cell carcinoma of the urinary bladder: Cytological aspect. J Cytol. 2014;31:50-52.

6. Jilla S, Prathipati A, Patnayak R, et al. Oesophageal malignancy with cutaneous metastasis over lower limb: Arare presentation. J Clin Sc Res. 2016;5:68-70.

7. Krathen RA, Orengo IF, Rosen T. Cutaneous metastasis: A meta-analysis of data. South Med J. 2003;96:164-166

\section{*Correspondence to:}

Rashmi Patnayak

Institute of Medical Sciences and SUM Hospital,

Odisha, India

E-mail: rashmipatnayak2002@yahoo.co.in 